\title{
Energy Upconversion in GaP/GaNP Core/Shell Nanowires for Enhanced Near-Infrared Light Harvesting
}

\author{
Alexandr Dobrovolsky, S. Sukrittanon, Y. J. Kuang, C. W. Tu, Weimin Chen and Irina \\ Buyanova
}

\section{Linköping University Post Print}

\section{Tweet}

N.B.: When citing this work, cite the original article.

Original Publication:

Alexandr Dobrovolsky, S. Sukrittanon, Y. J. Kuang, C. W. Tu, Weimin Chen and Irina Buyanova, Energy Upconversion in GaP/GaNP Core/Shell Nanowires for Enhanced NearInfrared Light Harvesting, 2014, Small, (10), 21, 4403-4408.

http://dx.doi.org/10.1002/smll.201401342

Copyright: Wiley-VCH Verlag http://www.wiley-vch.de/publish/en/

Postprint available at: Linköping University Electronic Press http://urn.kb.se/resolve?urn=urn:nbn:se:liu:diva-109929 


\section{WILEY-VCH}

DOI: 10.1002/(smll.20401342)

Article type: Full Paper

\section{Energy Upconversion in GaP/GaNP Core/Shell Nanowires for Enhanced Near-Infrared Light Harvesting}

Alexander Dobrovolsky, Supanee Sukrittanon, Yanjin Kuang, Charles W. Tu, Weimin M. Chen, and Irina A. Buyanova*

Dr. A. Dobrovolsky, Prof. W. M. Chen, Prof. I. A. Buyanova

Department of Physics, Chemistry and Biology

Linköping University

Linköping S-581 83, Sweden

E-mail: irb@ifm.liu.se

S. Sukrittanon

Graduate Program of Materials Science and Engineering

University of California, San Diego

La Jolla, California 92093, USA

Y. J. Kuang

Department of Physics

University of California, San Diego

La Jolla, California 92093, USA

Prof. C. W. Tu

Department of Electrical and Computer Engineering

University of California, San Diego

La Jolla, California 92093, USA

Keywords: nanowires, optical properties, photoluminescence, solar cells, upconversion

Semiconductor nanowires (NWs) have recently gained increasing interest due to their great potential for photovoltaics. A novel material system based on GaNP NWs is considered to be highly suitable for applications in efficient multi-junction and intermediate band solar cells. This work shows that though the bandgap energies of $\mathrm{GaN}_{\mathrm{x}} \mathrm{P}_{1-\mathrm{x}}$ alloys lie within the visible spectral range (i.e. within 540 - $650 \mathrm{~nm}$ for the currently achievable $\mathrm{x}<3 \%$ ), coaxial GaNP NWs grown on Si substrates can also harvest infrared light utilizing energy upconversion. This energy upconversion can be monitored via anti-Stokes near-band-edge photoluminescence (PL) from GaNP, visible even from a single NW. The dominant process 


\section{WILEY-VCH}

responsible for this effect is identified as being due to two-step two-photon absorption (TSTPA) via a deep level lying at about $1.28 \mathrm{eV}$ above the valence band, based on the measured dependences of the anti-Stokes PL on excitation power and wavelength. The formation of the defect participating in the TS-TPA process is concluded to be promoted by nitrogen incorporation. The revealed defect-mediated TS-TPA process can boost efficiency of harvesting solar energy in GaNP NWs, beneficial for applications of this novel material system in third-generation photovoltaic devices.

\section{Introduction}

Semiconductor nanowires (NWs) have recently emerged as a new class of materials with great potential as nano-building blocks for a wide variety of future applications ranging from sensors to optoelectronic and photonic devices. NWs are also considered as a very promising material system for the third-generation photovoltaic devices as nanowire architecture allows one to improve efficiency of harvesting solar energy while reducing fabrication costs as compared with bulk and thin-film photovoltaics. ${ }^{[1-4]}$ In this respect especially attractive are core/shell NWs as such radial design allows independent control of sizes, doping and compositions of core and shell layers, ${ }^{[5-7]}$ which can improve the efficiency of charge collection and optical absorption in vertically-aligned NW arrays. ${ }^{[8]} \mathrm{NW}$ solar cells based on a radial junction have been reported for several III-V compound semiconductors. ${ }^{[9-12]}$

The efficiency of NW-based solar cells made of a single semiconductor is limited by a narrow spectral range determined by the bandgap. Fabrication of multi-junction NW heterostructures using semiconductors with different energy gaps allows one to overcome this limitation, but the complexity of the structure and the device fabrication process remains a major disadvantage of this type of photovoltaic devices. An alternative approach that can extend the spectral range of light absorption while maintaining a simple single-junction design utilizes optical transitions via the so-called intermediate band (IB). ${ }^{[1315]}$ The IB 


\section{WILEY-VCH}

represents an energy band within the bandgap of a semiconductor that acts as an intermediate state for light absorption. This allows absorption of photons with energies lower than the bandgap energy to transfer electrons from the valence band (VB) to the conduction band (CB). The efficiency of such an IB solar cell is not restricted by the Shockley-Queisser limit for a single-junction solar cell and is predicted to reach as high as $63 \%$, i.e. similar to that of a triple-junction solar cell.

A novel material system attractive for applications in both multi-junction and IB solar cells is GaNP alloys. These alloys are obtained from parental gallium phosphide by incorporation of several percentages of nitrogen, which has profound effects on their lattice and energy structure. First of all, alloying of GaP with nitrogen reduces its lattice constant, thus minimizing (and event eliminating for some $\mathrm{N}$ compositions) lattice mismatch with Si. This is advantageous for lower cost photovoltaic devices since it allows epitaxial growth of GaNP on cheap Si substrates $^{[16,17]}$ and removes lattice matching constrains for GaNP/Si multi-junction structures. Secondly, a strong interaction between the N-related localized states and the extended states of the GaP host results in splitting of the CB states into two well separated subbands: $E^{+}$and $E^{-[18,19]}$ The lowest subband is narrow in energy and can act as IB. ${ }^{[14]}$ Energy positions of these subbands can be easily tuned as desired by choosing a proper alloy composition as an addition of $1 \%$ of nitrogen causes a down-shift of $\mathrm{E}^{-}$(upshift of $\mathrm{E}^{+}$) by about $0.1 \mathrm{eV} .^{[18,19]}$ Though fabrication processes of high-quality bulk and two-dimensional GaNP-based structures are well established, successful growth of this alloy in the NW geometry was achieved only most recently. ${ }^{[20]}$ The NW structures were concluded to have a high optical quality ${ }^{[21]}$ confirming their potential for future optoelectronic and photovoltaic applications.

The efficiency of solar energy harvesting can be further enhanced by introducing an additional IB due to a deep defect/impurity state that improves consumption of photons with energies below the bandgap of a light absorber. ${ }^{[22-25]}$ Such defect-mediated energy 


\section{WILEY-VCH}

upconversion was utilized in a variety of solar cell structures including dye-sensitized solar cells ${ }^{[23,24]}$ and ZnO-based UV photovoltaic cells, ${ }^{[25]}$ but was not explored so far in III-V based nanowires. In this work we show that energy upconversion exists in GaNP-based NWs and is promoted by nitrogen incorporation. We also demonstrate that this process extends the absorption range by about $0.7 \mathrm{eV}$ and identify the dominant mechanism responsible for this effect.

\section{Results and Discussion}

Figure 1 shows scanning electron microscopy (SEM) images of the studied NW arrays grown on Si substrates. In order to investigate impact of growth conditions and structural design on the energy upconversion, the following three types of structures were studied: (i) Ga(N)P NWs; (ii) GaP/GaNP and $\mathrm{Ga}_{1-\mathrm{x}} \mathrm{N}_{\mathrm{x}} \mathrm{P} / \mathrm{Ga}_{1-\mathrm{y}} \mathrm{N}_{\mathrm{y}} \mathrm{P}$ core/shell $\left(\mathrm{x}<\mathrm{y}\right.$ ) structures; and (iii) $\mathrm{GaP} / \mathrm{Ga}_{1-}$ ${ }_{x} \mathrm{~N}_{\mathrm{x}} \mathrm{P} / \mathrm{Ga}_{1-\mathrm{y}} \mathrm{N}_{\mathrm{y}} \mathrm{P}$ core/shell/shell NWs $(\mathrm{x}>\mathrm{y})$ with the outer passivating shell. As obvious from Figure 1, the NWs are hexagonal in their cross sections, indicating that they were epitaxially grown following the Si [111] crystal orientation. They are rather uniform in sizes and have an axial length of about 2 - $2.5 \mu \mathrm{m}$. In order to perform optical characterization of single NWs, some wires were mechanically transferred onto another Si substrate. Representative SEM images of such individual GaP and GaP/GaNP NWs are shown in Figures 2a and 2b, respectively.

Representative photoluminescence (PL) spectra measured from the investigated structures under the above bandgap excitation are shown in Figure 2c, taking as an example individual $\mathrm{GaP}$ and $\mathrm{GaP} / \mathrm{GaNP}$ NWs. In the case of the GaP NW, the PL spectrum contains a series of sharp lines within the 540-590 nm spectral range that can be attributed to recombination of excitons bound to residual impurities and/or defect centers. ${ }^{[26]}$ The near-band-edge emission of the GaNP-based NWs, on the other hand, is dominated by a broad asymmetric PL band that originates from radiative recombination of excitons trapped at various $\mathrm{N}$-related localized 


\section{WILEY-VCH}

states in the GaNP alloy. ${ }^{[21,27]}$ The observed gradual redshift of this PL emission with increasing $\mathrm{N}$ content reflects the giant bowing in the bandgap energy of GaNP ${ }^{[18]}$. This is accompanied by an overall PL increase with increasing N, due to an increasing number of emitting centers and a change in the bandgap character from an indirect one in $\mathrm{GaP}$ to a quasidirect bandgap in GaNP alloys with $[\mathrm{N}]>0.4 \%{ }^{[18,27]}$ In the case of GaP/Ga ${ }_{1-\mathrm{x}} \mathrm{N}_{\mathrm{x}} \mathrm{P} / \mathrm{Ga}_{1-\mathrm{y}} \mathrm{N}_{\mathrm{y}} \mathrm{P}$ structures, the PL transitions occur in the inner $\mathrm{Ga}_{1-\mathrm{x}} \mathrm{N}_{\mathrm{x}} \mathrm{P}$ shell that has the smallest bandgap energy, due to fast trapping of photoexcited carriers from the adjacent $\mathrm{GaP}$ core and the outer $\mathrm{Ga}_{1-\mathrm{y}} \mathrm{N}_{\mathrm{y}} \mathrm{P}$ shell with wider bandgaps. Very similar PL spectra were also detected from the NW arrays, indicating high optical quality and uniformity of the investigated structures.

Most remarkably, the same near-band-edge PL emission of GaNP can also be detected when the excitation photon energy is tuned below the alloy's bandgap providing solid evidence for an energy upconversion process. Spectra of the upconverted PL (UPL) emission are shown in Figure $2 \mathrm{~d}$ and are identical to those measured under the above bandgap excitation - see Figure 2c. An average UPL efficiency for a single GaP/GaNP NW with [N] = $0.9 \%$ under excitation density of $10^{4} \mathrm{~W} / \mathrm{cm}^{-2}$ and $\lambda_{\text {exc }}=820$ can be estimated as $0.2 \%$. The revealed upconversion process only occurs in GaNP, as it is observed in all NWs that contain GaNP but could not be detected from the GaP NWs, judging from a lack of the upconverted PL in these structures despite of a reasonably high PL intensity under the above bandgap excitation.

In order to gain detailed information on the mechanism responsible for the observed energy upconversion, we first analyze its dependences on a photo-excitation power $\left(\mathrm{W}_{\text {exc }}\right)$. The UPL intensity ( $\left.\mathrm{I}_{\text {upl }}\right)$ is found to be proportional to $\mathrm{W}_{\text {exc }}{ }^{\mathrm{n}}$, where the power factor $\mathrm{n}$ is close to 1.3 and does not depend on $\mathrm{N}$ composition of the GaNP shell. This is illustrated in Figure 3a, taking as an example results obtained from an array of $\mathrm{GaP} / \mathrm{GaN}_{0.009} \mathrm{P}_{0.991} \mathrm{NWs}$ under below bandgap excitation with wavelengths $\lambda_{\text {exc }}=820,870$ and $950 \mathrm{~nm}$. On the other hand, linear dependence between PL intensity and $\mathrm{W}_{\mathrm{exc}}$, which is typical for excitonic transitions, 


\section{WILEY-VCH}

was observed when the excitation photon energy was tuned above the bandgap of GaNP, e.g. for $\lambda_{\text {exc }}=532 \mathrm{~nm}$.

Common mechanisms for energy upconversion in semiconductor materials that lead to generation of non-equilibrium carriers under below-bandgap excitation include two-photon absorption (TPA) and two-step two-photon absorption (TS-TPA). ${ }^{[28-31]}$ In both processes excitation of carriers is accomplished by sequential absorption of two photons via an intermediate state. In the TPA process, the involved intermediate state is a virtual one which leads to quadratic dependence of the UPL intensity on $\mathrm{W}_{\text {exc }}$ and makes this process efficient only under very high excitation powers. On the other hand, the photon absorption during the TS-TPA process is mediated by a real state, e.g., a deep impurity or defect level. This results in a substantially higher efficiency of this process at low $\mathrm{W}_{\mathrm{exc}}$ and its weaker (i.e. with the power factor $n$ below 2) dependence on the excitation power, provided that the involved intermediate state is long lived. The subquadratic power dependence of the UPL emission in the investigated GaNP-based NWs, therefore, implies that the dominant energy upconversion process in these structures is the TS-TPA via an impurity or defect level.

The corresponding absorption steps that lead to generation of non-equilibrium carriers are shown schematically in the inset in Figure 3b (the red arrows). They include excitation of an electron from the VB to the deep level (DL) leaving a hole in the VB, and excitation of the electron from this level to the CB. The created electron-hole pairs are then trapped by the Nrelated centers giving rise to the near-band-edge emission. The energy position of the involved deep level can be determined by studying spectral dependence of TS-TPA based on excitation spectroscopy of the upconverted PL. Such UPL excitation (UPLE) spectra measured from a single and an ensemble of GaP/ $\mathrm{Ga}_{1-\mathrm{x}} \mathrm{N}_{\mathrm{X}} \mathrm{P}$ NWs with different $\mathrm{N}$ contents are shown in Figure 3b by symbols and solid lines, respectively, revealing a clear threshold at around 960 - $970 \mathrm{~nm}(1.28-1.29 \mathrm{eV})$. The monitored threshold of the TS-TPA could in principle be related to either the $\mathrm{VB} \rightarrow \mathrm{DL}$ or $\mathrm{DL} \rightarrow \mathrm{CB}$ absorption transitions. Increasing $\mathrm{N}$ 


\section{WILEY-VCH}

content in the GaNP alloy is not expected to affect the VB position (at least within the studied range of nitrogen compositions) but is known to cause a strong downshift of the CB edge. ${ }^{[32]}$ For the GaNP NWs shown in Figure 3b, the change in N content from 0.9 to $2.2 \%$ should result in a CB downshift of around $0.12 \mathrm{eV} .{ }^{[18,19]}$ Since energy positions of deep levels in semiconductors are usually pinned to the vacuum level, ${ }^{[33]}$ a CB downshift should lead to a comparable change of the threshold energy for the DL $\rightarrow$ CB transition. In sharp contrast, only a marginal change is expected for the $\mathrm{VB} \rightarrow \mathrm{DL}$ absorption. Our experimental finding that the threshold energy does not depend on the $\mathrm{N}$ composition in the alloy as shown in Figure 3b implies that it is related to the absorption process $\mathrm{VB} \rightarrow \mathrm{DL}$. The observed dependence of the UPL efficiency on the excitation energy reflects spectral dependence of the optical crosssection of this transition. By analyzing this spectral dependence using the formalism developed in Ref. 34, the energy level of the deep state acting as a stepping stone for TS-TPA can then be estimated as being at around $1.28 \pm 0.2 \mathrm{eV}$ above the VB edge.

Let us now briefly discuss the origin of the involved defect. The lack of energy upconversion in the GaP NWs unambiguously proves that the defect formation is favored in the GaNP alloy. So far, defect properties of this material in the NW morphology are largely unknown. The only identified defect is a complex involving a $\mathrm{P}_{\mathrm{Ga}}$ antisite or a $\mathrm{P}_{\mathrm{i}}$ interstitial atom that was shown to act as an important non-radiative recombination center in $\mathrm{GaP} / \mathrm{GaNP}$ core/shell nanowires, based on optically detected magnetic resonance (ODMR) studies. ${ }^{[21]}$ The same defect, labeled as DD1, was previously observed in GaNP epilayers at the interface with a GaP substrate. ${ }^{[35]}$ We have shown that in GaNP-based NWs, the DD1 defect is preferably formed either at the GaNP/GaP interface or at the surface of the GaNP shell. ${ }^{[35]}$ The upconversion efficiency, however, seems to be unaffected by the presence of the GaNP/GaP interface or conditions at the GaNP surface as it remains rather similar in all GaNP-based NWs including: (i) GaNP NWs (i.e. without the GaNP/GaP interface but with unprotected GaNP surface); (ii) GaP/GaNP core/shell structures (i.e. with the GaNP/GaP 


\section{WILEY-VCH}

interface and unprotected GaNP surface) and (iii) $\mathrm{GaP} / \mathrm{Ga}_{1-\mathrm{x}} \mathrm{N}_{\mathrm{x}} \mathrm{P} / \mathrm{Ga}_{1-\mathrm{y}} \mathrm{N}_{\mathrm{y}} \mathrm{P}$ core/shell/shell NWs with the optically-active inner $\mathrm{Ga}_{1-\mathrm{x}} \mathrm{N}_{\mathrm{x}} \mathrm{P}$ shell (i.e. with the GaP/GaNP interface and protected surface). This makes unlikely involvement of the surface- and interface-related defects in the energy upconversion and suggests the defect formation most likely occurs in bulk regions of GaNP NWs. The formation process is also found to be insensitive to the GaNP growth mode, as the upconversion efficiency is found to be very similar in the GaNP NWs grown via the vapor-liquid-solid (VLS) mechanism and in the GaP/GaNP core/ shell structures where growth of the shell layer was step-mediated. It is also of the same order as that measured from the reference GaNP epilayers grown on GaP substrates. Common grownin defects in bulk GaNP epilayers and two-dimensional quantum well structures include deep $\mathrm{N}$-related centers, e.g. N-related clusters and N-N split interstitials, ${ }^{[27,37-39]}$ and complex defects containing a Ga interstitial $\left(\mathrm{Ga}_{\mathrm{i}}\right)$ atom at their core. ${ }^{[40-42]}$ All of them introduce deep states in the GaNP bandgap and can, therefore, be potentially important for the energy upconversion. Further studies are required to clarify this important issue.

The remaining question is the origin of photons participating in TS-TPA. The first photon participating in this process is provided by an excitation source, i.e. a laser in our experiments. The second photon, on the other hand, may be supplied either by the laser light or by other radiative recombination channels via photon recycling. ${ }^{[30,43-45]}$ In the latter case, a slow rising of the UPL signal which persists after the end of the laser pulse due to a slow feeding via photon recycling is usually observed. In order to evaluate importance of photon recycling in the studied structures, we have performed time-resolved PL measurements under above and below bandgap excitation. Representative transients of the near-band-edge PL emission measured under such conditions from the GaP/GaNP core/shell NWs are shown in Figure 3c. The PL decays are relatively slow ( $\tau>10 \mathrm{ns)} \mathrm{and} \mathrm{are} \mathrm{likely} \mathrm{determined} \mathrm{by} \mathrm{the} \mathrm{radiative}$ lifetime of excitons trapped at the N-related localized states as the determined lifetime is of the same order as that previously determined in the GaNP alloys. ${ }^{[27,46]}$ Most importantly, the 


\section{WILEY-VCH}

PL rising time remains the same under one-and two-photon excitation, which rules out significant contributions from the photon recycling in the upconversion process and implies that both photons participating in the TS-TPA are provided by the laser.

\section{Conclusion}

In conclusion, we have shown that GaNP NWs and related coaxial structures exhibit energy upconversion, evident from the appearance of the near-band-edge PL emission within the visible spectral range under below bandgap excitation by near-infrared light. The process responsible for the upconversion is assigned to two-step two-photon absorption mediated by defect states and does not require photon recycling, based on the measured dependence of the upconverted PL on the excitation power as well as on the results from the UPL transient measurements. From photoluminescence excitation of the upconverted emissions, the energy position of this intermediate defect state is determined as being at around $\mathrm{E}_{\mathrm{v}}+1.28 \mathrm{eV}$. This means that TS-TPA extends the range of light harvesting in GaNP NWs by about $0.7 \mathrm{eV}$. The responsible defect is concluded to be formed within the bulk regions of GaNP NWs and either directly involves a $\mathrm{N}$ atom(s) or is promoted by the $\mathrm{N}$ presence in the alloy. The revealed defect-mediated TS-TPA process can boost efficiency of harvesting solar energy in GaNP NWs, beneficial for applications of this novel material system in third-generation photovoltaic devices.

\section{Experimental Section}

All NW structures studied in this work were grown by gas-source molecular beam epitaxy (GS MBE) on (111)-oriented Si substrates using as a catalyst Ga droplets formed on the substrate surface. The following types of structures were studied: (i) GaP and GaNP NWs with diameters ranging between 80 and $100 \mathrm{~nm}$; (ii) GaP/GaNP and $\mathrm{Ga}_{1-\mathrm{x}} \mathrm{N}_{\mathrm{x}} \mathrm{P} / \mathrm{Ga}_{1-\mathrm{y}} \mathrm{N}_{\mathrm{y}} \mathrm{P}$ core/shell $(\mathrm{x}<\mathrm{y})$ structures having a 50-nm thick shell layer; and (iii) GaP/Ga $\mathrm{Ga}_{1-\mathrm{x}} \mathrm{N}_{\mathrm{x}} \mathrm{P} / \mathrm{Ga}_{1-\mathrm{y}} \mathrm{N}_{\mathrm{y}} \mathrm{P}$ 


\section{WILEY-VCH}

core/shell/shell NWs $(\mathrm{x}>\mathrm{y})$ with the outer passivating shell of 30-80 nm. The uniform GaP and GaNP NWs were grown under the VLS mechanism at substrate temperatures $T_{\text {sub }}$ of 580 $600{ }^{\circ} \mathrm{C}$ and $615{ }^{\circ} \mathrm{C}$, respectively. The V/III incorporation ratio was kept at around $1.5-2.0$ during the GaP growth but was increased to 2.5 with a nitrogen plasma ignited for the growth of GaNP. On average, an axial growth rate of GaP and GaNP NWs was of $\sim 78 \mathrm{~nm} /$ minute $\sim 150 \mathrm{~nm} /$ minute, respectively. ${ }^{[20]}$ The same growth conditions were also used for the core layers in (ii) and (iii) structures. The GaNP shells were fabricated via the step-mediated growth performed at a lower $\mathrm{T}_{\text {sub }}$ of $450-510{ }^{\circ} \mathrm{C}$ with a V/III incorporation ratio of 3.5 - 4.5 and a radial growth rate of around $1.8 \mathrm{~nm} / \mathrm{min}$. Nitrogen composition in the shell layers was varied by changing the power of rf-plasma and a $\mathrm{N}$ flux and was estimated ${ }^{[20]}$ from the roomtemperature photoluminescence data according to the band anticrossing model. ${ }^{[18]}$ The details of the NW growth process can be found in Ref. 20. Structural characterization of the NWs was performed by scanning electron microscopy (SEM). It was found that NWs are hexagonal in shape, indicating that they were epitaxially grown following the Si [111] crystal orientation. They are rather uniform in sizes and have an axial length of about 2 - $2.5 \mu \mathrm{m}$. In order to perform optical characterization of individual NWs, some wires were mechanically transferred onto another Si substrate. Representative SEM images of such individual GaP and GaP/GaNP NWs are shown in Figures 2a and 2b, respectively.

Micro- photoluminescence ( $\mu$-PL) spectroscopy of individual NWs was carried out using a Horiba Jobin Yvon HR800 spectrometer with a CCD detector. The PL spectra from an ensemble of NWs were detected by a photomultiplier tube after being dispersed by a doublegrating monochromator. Continuous wave (cw) PL was excited by using the $445 \mathrm{~nm}$ line of a diode laser or the $532 \mathrm{~nm}$ line of a solid-state laser for excitation above the bandgap of GaNP and a tunable Ti:sapphire solid state laser for excitation below the bandgap. The latter was also employed as an excitation source in the excitation measurements of the anti-stokes (or upconverted) PL. In order to avoid contributions of the laser leakage in the measured PL 


\section{WILEY-VCH}

spectra, a long-pass (short-pass) optical filter was inserted in the excitation (detection) path. Time-resolved PL measurements were performed using a pulsed Ti:sapphire laser with a pulse duration of 2 ps and a repetition rate of $76 \mathrm{MHz}$. Transient PL signals were detected by a streak camera combined with a grating monochromator. All optical measurements were performed at $5 \mathrm{~K}$.

\section{Acknowledgements}

Financial support by the Swedish Research Council (grant no. 621-2010-3815) is greatly appreciated. The nanowire growth is supported by the National Science Foundation under Grant Nos. DMR-0907652, DMR-1106369 and the Royal Government of Thailand Scholarship (DPST). The authors also thank S. L. Chen for his help with time-resolved PL measurements.

Received: ((will be filled in by the editorial staff))

Revised: ((will be filled in by the editorial staff)) Published online: ((will be filled in by the editorial staff))

Nature 2007, 449, 885.

[2] A. I. Hochbaum, P. D. Yang, Chem. Rev. 2010, 110, 527.

[3] E. C. Garnett, M. L. Brongersma, Y. Cui, M. D. McGehee, Annu. Rev. Mater. Res. 2011, 41, 269.

[4] J. Wallentin, N. Anttu, D. Asoli, M. Huffman, I. Åberg, M. H. Magnusson, G. Siefer,

P. Fuss-Kailuweit, F. Dimroth, B. Witzigmann, H. Q. Xu, L. Samuelson, K. Deppert, M. T. Borgström, Science 2013, 339, 1057.

[5] H. Goto, K. Nosaki, K. Tomioka, S. Hara, K. Hiruma, J. Motohisa, T. Fukui, Appl. Phys. Express 2009, 2, 035004.

[6] B. Tian, T. J. Kempa, C. M. Lieber, Chem. Soc. Rev. 2009, 38, 16.

[7] S. Kumar, M. Jones, S. S. Lo, G. D. Scholes, Small 2007, 3, 1633.

[8] B. M. Kayes, H. A. Atwater, N. S. Lewis, J. Appl. Phys. 2005, 97, 114302. 


\section{WILEY-VCH}

[9] C. Colombo, M. Heiß, M. Graetzel, A. Fontcuberta i Morral, Appl. Phys. Lett. 2009, 94, 173108.

[10] Y. Dong, B. Tian, T. J. Kempa, C. M. Lieber, Nano Lett. 2009, 9, 2183.

[11] M. Heurlin, P. Wickert, S. Fält, M. T. Borgström, K. Deppert, L. Samuelson, M. H. Magnusson, Nano Lett. 2011, 11, 2028.

[12] M. Tchernycheva, L. Rigutti, G. Jacopin, A. de Luna Bugallo, P. Lavenus, F. H. Julien, M. Timofeeva, A. D. Bouravleuv, G. E. Cirlin, V. Dhaka, H. Lipsanen, L. Largeau, Nanotechnology 2012, 23, 265402.

[13] A. Luque, A. Martí, Phys. Rev. Lett. 1997, 78, 5014.

[14] N. López, L. A. Reichertz, K. M. Yu, K. Campman, W. Walukiewicz, Phys. Rev. Lett. 2011, 106, 028701.

[15] A. Luque, A. Martí, C. Stanley, Nature Photonics 2012, 6, 146.

[16] H. Yonezu, Semicond. Sci. Technol. 2002, 17, 762.

[17] I. A. Buyanova, W. M. Chen, C. W. Tu, Semicond. Sci. Technol. 2002, 17, 815.

[18] W. Shan, W. Walukiewicz, K. M. Yu, J. Wu, J. W. Ager III, E. E. Haller, H. P. Xin, C. W. Tu, Appl. Phys. Lett. 2000, 76, 3251.

[19] I. A. Buyanova, M. Izadifard, A. Kasic, H. Arwin, W. M. Chen, H. P. Xin, Y. G. Hong; C. W. Tu, Phys. Rev. B 2004, 70, 085209.

[20] Y. J. Kuang, S. Sukrittanon, H. Li, C. W. Tu, Appl. Phys. Lett. 2012, 100, 053108.

[21] A. Dobrovolsky, J. E. Stehr, S. L. Chen, Y. J. Kuang, S. Sukrittanon, C. W. Tu, W. M. Chen, I. A. Buyanova, Appl. Phys. Lett. 2012, 101, 163106.

[22] T. Trupke, M. A. Green, P. Würfel, J. Appl. Phys. 2002, 92, 4117.

[23] G.-B. Shan, G. P. Demopoulos, Adv. Mater. 2010, 22, 4373.

[24] W. Zou, C. Visser, J. A. Maduro, M. S. Pshenichnikov, J. C. Hummelen, Nature Photonics 2012, 6, 560.

[25] J. J. Cole, X. Wang, R. J. Knuesel, H. O. Jacobs, Nano Lett. 2008, 8, 1477. 


\section{WILEY-VCH}

[26] P. J. Dean, D. G. Thomas, C. J. Frosch, J. Phys C: Solid State Phys. 1984, 17, 747.

[27] I. A. Buyanova, G. Pozina, J. P. Bergman, W. M. Chen, H. P. Xin, C. W. Tu, Appl. Phys. Lett. 2002, 81, 52.

[28] B. Clerjaud, F. Gendron, C. Porte, Appl. Phys. Lett. 1981, 38, 212.

[29] P. P. Paskov, P. O. Holtz, B. Monemar, J. M. Garcia, W. V. Schoenfeld, P. M. Petroff, Appl. Phys. Lett. 2000, 77, 812.

[30] M. Izadifard, J. P. Bergman, W. M. Chen, I. A. Buyanova, Y. G. Hong, C. W. Tu, J. Appl. Phys. 2006, 99, 073515.

[31] J. E. Stehr, S. L. Chen, N. Koteeswara Reddy, C. W. Tu, W. M. Chen, I. A. Buyanova, Adv. Funct. Mater., DOI: 10.1002/adfm.201400220.

[32] L. Bellaiche, S.-H. Wei, A. Zunger, Phys. Rev. B 1996, 54, 17568.

[33] C. G. Van de Walle, J. Neugebauer, Nature 2003, 423, 626.

[34] G. Lucovsky, Solid State Communications 1965, 3, 299.

[35] D. Dagnelund, I. P. Vorona, L. S. Vlasenko, X. J. Wang, A. Utsumi, Y. Furukawa, A. Wakahara, H. Yonezu, I. A. Buyanova, W. M. Chen, Phys. Rev. B 2010, 81, 115334.

[36] J. E. Stehr, A. Dobrovolsky, P. Persson, S. Sukrittanon, Y. J. Kuang, C. W. Tu, W. M. Chen, I. A. Buyanova, unpublished work.

[37] J. N. Baillargeon, K. Y. Cheng, G. E. Hofler, P. J. Pearah, K. C. Hsieh, Appl. Phys. Lett. 1992, 60, 2540.

[38] P. R. C. Kent, A Zunger, Phys. Rev. B 2001, 64, 115208.

[39] S. B. Zhang, S. H. Wei, Phys. Rev. Lett. 2001, 86, 1789.

[40] N. Q. Thinh, I. P. Vorona, I. A. Buyanova, W. M. Chen, S. Limpijumnong, S. B.

Zhang, Y. G. Hong, H. P. Xin, C. W. Tu, A. Utsumi, Y. Furukawa, S. Moon, A. Wakahara, H. Yonezu Phys. Rev. B 2005, 71, 125209.

[41] A. F. Basile, S. Hatakenaka, H. Okada, A. Wakahara, J. Vac. Sci. Technol. A 2009, 27, 531. 


\section{WILEY-VCH}

[42] D. Dagnelund, I. A. Buyanova, X. J. Wang, W. M. Chen, A. Utsumi, Y. Furukawa, A.

Wakahara, H. Yonezu, J. Appl. Phys. 2008, 103, 063519.

[43] R. Hellmann, A. Euteneuer, S. G. Hense, J. Feldmann, P. Thomas, E. O. Göbel, D. R.

Yakovlev, A. Wagg, G. Landwehr, Phys. Rev. B 1995, 51, 18053.

[44] H. M. Cheong, B. Fluegel, M. C. Hanna, A. Mascarenhas, Phys. Rev. B 1998, 58, R4254.

[45] S. L. Chen, J. Stehr, N. Koteeswara Reddy, C. W. Tu, W. M. Chen, I. A. Buyanova, Appl. Phys. B 2012, 108, 919.

[46] A. Dobrovolsky, S. L. Chen, Y. J. Kuang, S. Sukrittanon, C. W. Tu, W. M. Chen, I. A. Buyanova, Nanoscale Res. Lett. 2013, 8, 239.

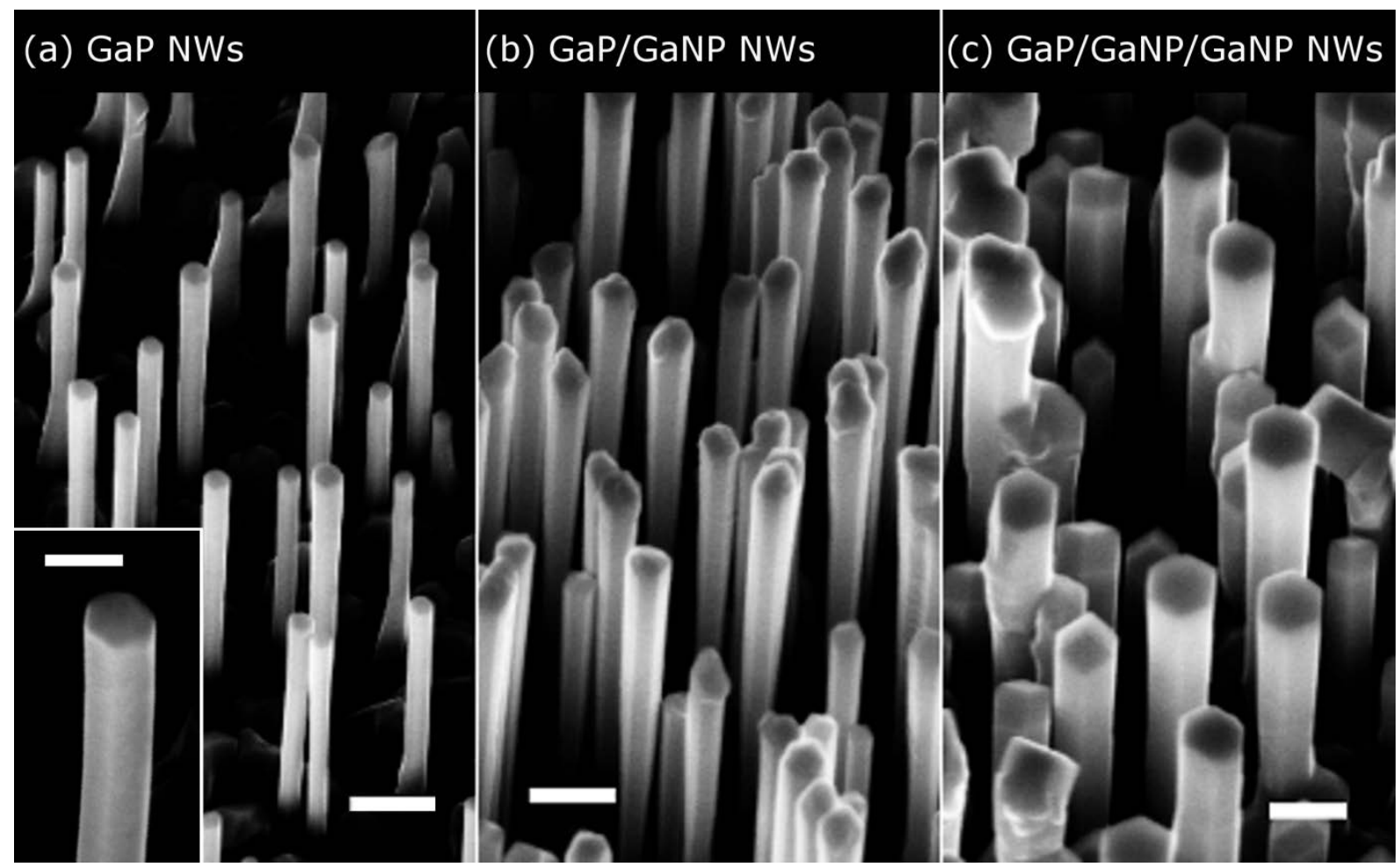

Figure 1. SEM images of GaP NWs (a), GaP/GaNP NWs (b) and GaP/GaNP/GaNP NWs (c). The scale bar is $300 \mathrm{~nm}$. (The scale bar in the inset is $100 \mathrm{~nm}$.) 

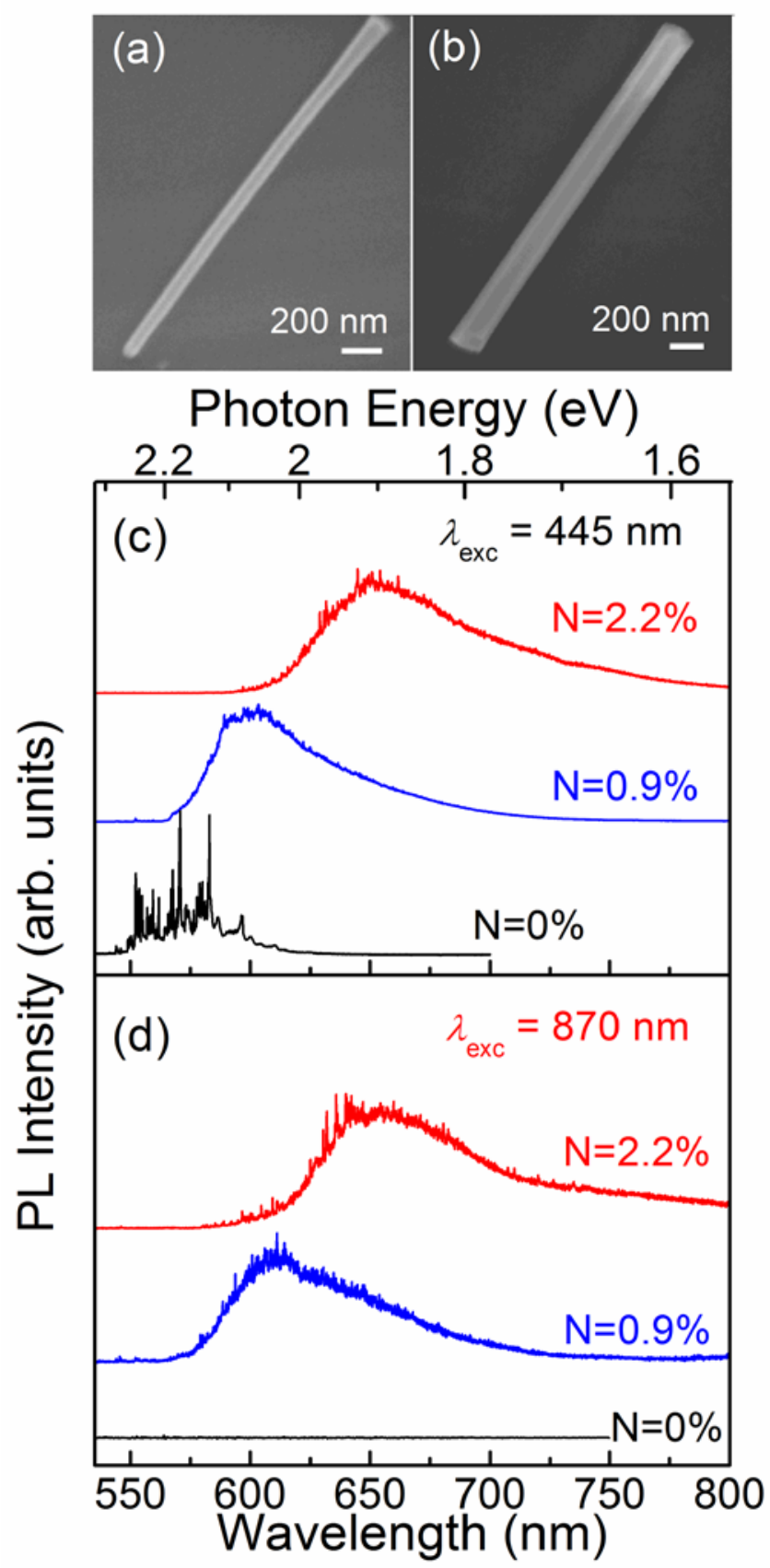

Figure 2. (a-b) Representative SEM images of a GaP NW (a) and GaP/GaNP core/shell NW (b) transferred onto a Si substrate (top view). (c-d) PL spectra from the single GaP NW ( $\mathrm{N}=0 \%$ ) and $\mathrm{GaP} / \mathrm{GaNP}$ core/shell NW with $\mathrm{N}=0.9$ and $2.2 \%$ measured at $5 \mathrm{~K}$ under above (c) and below (d) bandgap excitation conditions. The spectra are normalized to the same peak intensity and are vertically shifted for clarity. 

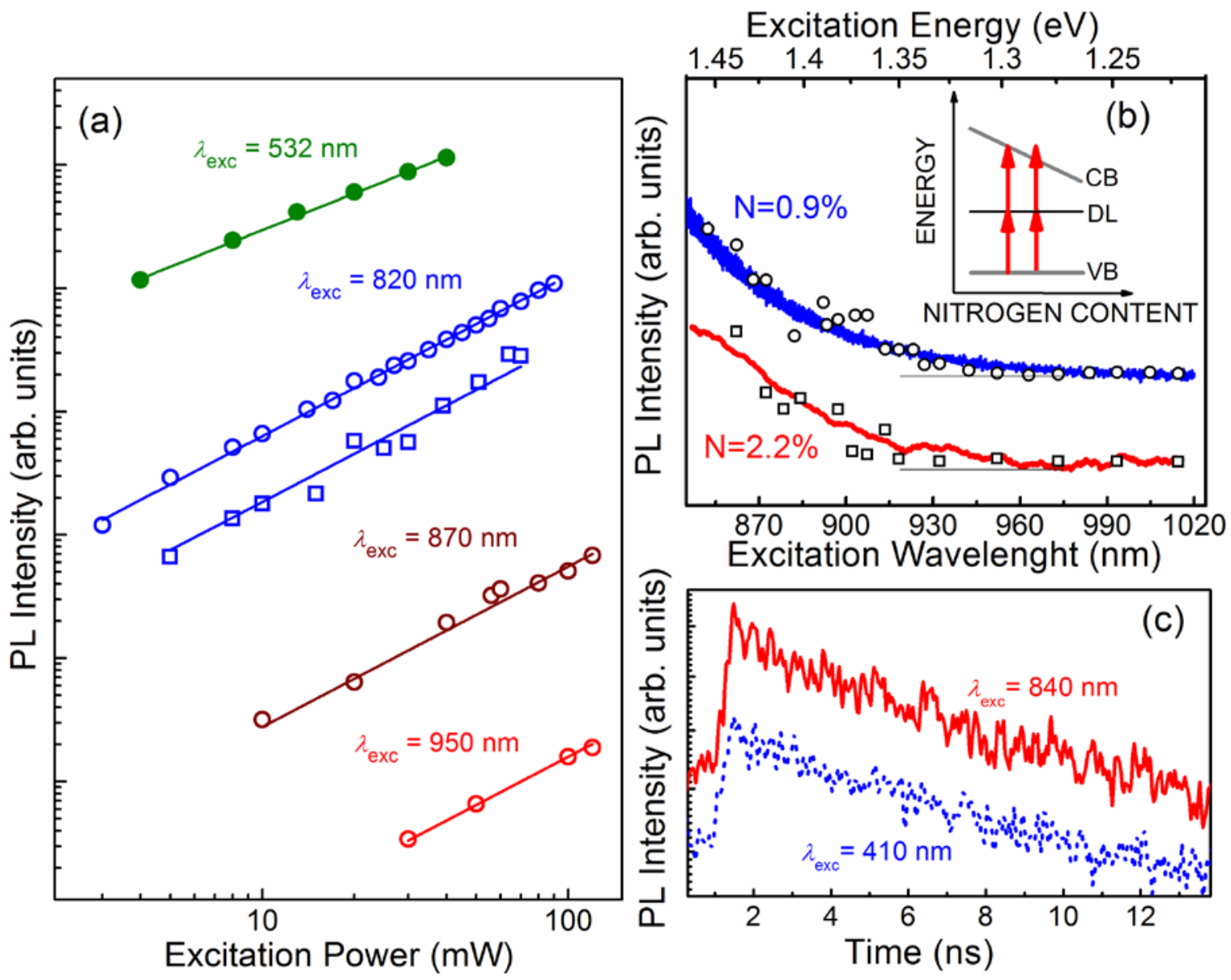

Figure 3. (a) Excitation power dependences of the UPL intensity measured from vertical arrays of GaP/GaNP core/shell NWs at the specified excitation photon wavelength under continuous-wave (circles) and pulsed (open squares) excitation. The solid lines represent the best fit of the data by the power functions as specified in the text. (b) Excitation spectra of the UPL emission measured from the GaP/GaNP NW arrays with the specified N compositions (solid lines) and single NWs with the same $\mathrm{N}$ content (symbols). The detection energies in the UPLE measurements were set at the peak position of the UPL emission. The spectra are vertically offset for clarity. The zero PL intensity for each spectrum is indicated by the solid line. The inset shows a schematic diagram of the upconversion processes. The red vertical arrows denote the TS-TPA process via the deep level (DL). (c) PL decays measured from the $\mathrm{GaP} / \mathrm{GaNP}$ NWs under one-photon (the dashed line) and two-photon (the solid line) excitation. 


\section{WILEY-VCH}

GaNP nanowires represent a novel material system attractive for applications in advanced multi-junction and intermediate band solar cells. The spectral range for light absorption in this system can be engineered utilizing energy upconversion via intrinsic defects induced by alloying with nitrogen. This finding paves the way for improving efficiency of photovoltaic devices based on defect engineering.

Keywords: nanowires, optical properties, photoluminescence, solar cells, upconversion

A. Dobrovolsky, S. Sukrittanon, Y. J. Kuang, C. W. Tu, W. M. Chen, I. A. Buyanova*

Energy Upconversion in GaP/GaNP Core/Shell Nanowires for Enhanced Near-Infrared Light Harvesting

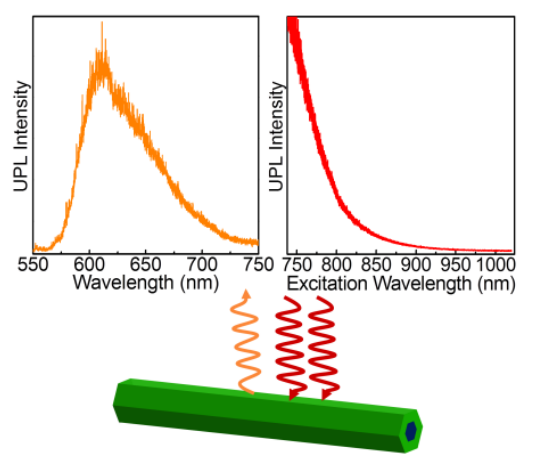

